VERSITA

Journal of Applied Mathematics, Statistics and Informatics (JAMSI), 8 (2012), No. 2

\title{
SEQUENCES OF INEQUALITIES AMONG DIFFERENCES OF GINI MEANS AND DIVERGENCE MEASURES
}

INDER J. TANEJA

\begin{abstract}
In 1938, Gini [3] studied a mean having two parameters. Later, many authors studied properties of this mean. In particular, it contains the famous means as harmonic, geometric, arithmetic, etc. Here we considered a sequence of inequalities arising due to particular values of each parameter of Gini's mean. This sequence generates many nonnegative differences. Not all of them are convex. We have established new sequences of inequalities of these differences. Some refinement inequalities are also presented. Considering in terms of probability distributions these differences, we have made connections with some well known divergence measures.
\end{abstract}

Mathematics Subject Classification 2000: 94A17; 26A48; 26D07

Additional Key Words and Phrases: Arithmetic mean; Geometric Mean; Harmonic Mean; Gini Mean; Power Mean; Differences of Means; Divergence measures

\section{GINI MEAN OF ORDER $R$ AND $S$}

The Gini [3] mean of order $r$ and $s$ is given by

$$
E_{r, s}(a, b)=\left\{\begin{array}{ll}
\left(\frac{a^{r}+b^{r}}{a^{s}+b^{s}}\right)^{\frac{1}{r-s}} & r \neq s \\
\exp \left(\frac{a^{r} \ln a+b^{r} \ln b}{a^{r}+b^{r}}\right) & r=s \neq 0 . \\
\sqrt{a b} & r=s=0
\end{array} .\right.
$$

In particular when $s=0$ in (1), we have

$$
E_{r, 0}(a, b):=B_{r}(a, b)=\left\{\begin{array}{ll}
\left(\frac{a^{r}+b^{r}}{2}\right)^{\frac{1}{r}}, & r \neq 0 \\
\sqrt{a b}, & r=0
\end{array} .\right.
$$

Again, when $s=r-1$ in (1), we have

$$
E_{r, r-1}(a, b):=K_{s}(a, b)=\frac{a^{r}+b^{r}}{a^{r-1}+b^{r-1}}, r \in \mathrm{R} .
$$

The expression (2) is famous as mean of order $r$ or power mean. The expression (3) is known as Lehmer mean[4]. Both these means are monotonically increasing in $r$. Moreover, these two have the following inequality [2] among each other:

$$
B_{r}(a, b)\left\{\begin{array}{ll}
<L_{r}(a, b), & r>1 \\
>L_{r}(a, b), & r<1
\end{array} .\right.
$$

DOI 10.2478/v10294-012-0014-2

CUniversity of SS. Cyril and Methodius in Trnava 
Since $E_{r, s}=E_{s, r}$, the Gini-mean $E_{r, s}(a, b)$ given by (1) is an increasing function in $r$ or $s$. Using the monotonicity property [1], [7], [5] we have the following inequalities:

$$
\begin{aligned}
E_{-3,-2} \leq & E_{-2,-1} \leq E_{-3 / 2,-1 / 2} \leq E_{-1,0} \leq E_{-1 / 2,0} \leq \\
& \leq E_{-1 / 2,1 / 2} \leq E_{0,1 / 2} \leq E_{0,1} \leq E_{0,2} \leq E_{1,2}
\end{aligned}
$$

or

$$
\begin{aligned}
E_{-3,-2} \leq & E_{-2,-1} \leq E_{-3 / 2,-1 / 2} \leq E_{-1,0} \leq E_{-1 / 2,0} \leq \\
& \leq E_{-1 / 2,1 / 2} \leq E_{0,1 / 2} \leq E_{0,1} \leq E_{1 / 2,1} \leq E_{1,2} .
\end{aligned}
$$

Equivalently,

$$
\begin{aligned}
K_{-2} \leq & K_{-1} \leq K_{-1 / 2} \leq\left(K_{0}=B_{-1}=H\right) \leq B_{-1 / 2} \leq \\
& \leq\left(K_{1 / 2}=B_{0}=G\right) \leq B_{1 / 2} \leq\left(K_{1}=B_{1}=A\right) \leq\left(B_{2}=S\right) \leq K_{2},
\end{aligned}
$$

or

$$
\begin{aligned}
K_{-2} \leq & K_{-1} \leq K_{-1 / 2} \leq\left(K_{0}=B_{-1}=H\right) \leq B_{-1 / 2} \leq \\
& \leq\left(K_{1 / 2}=B_{0}=G\right) \leq B_{1 / 2} \leq\left(K_{1}=B_{1}=A\right) \leq E_{1 / 2,1} \leq K_{2} .
\end{aligned}
$$

where $H, G, A$ and $S$ are respectively, the harmonic, geometric, arithmetic and the square-root means. We observe from the expression (7) that the means considered either are the particular cases of (2) or of (3), while in the second case we have we have a mean $E_{1 / 2,1}$, which is neither a particular case of (2) nor of (3). We can easily find values proving that there is no relation between $S$ and $E_{1 / 2,1}$. Summarizing the expressions (7) and (8), we can write them in joint form as

$$
P_{1} \leq P_{2} \leq P_{3} \leq H \leq P_{4} \leq G \leq N_{1} \leq A \leq\left(P_{5} \text { or } S\right) \leq P_{6},
$$

where $P_{1}=K_{-2}, P_{2}=K_{-1}, P_{3}=K_{-1 / 2}, P_{4}=B_{-1 / 2}, N_{1}=B_{1 / 2}, P_{5}=E_{1 / 2,1}$ and $P_{5}=K_{2}$.

In $[9 ;$ ?], the author studied the following inequalities:

$$
H \leq G \leq N_{1} \leq N_{3} \leq N_{2} \leq A \leq S,
$$

where

$$
N_{2}(a, b)=\left(\frac{\sqrt{a}+\sqrt{b}}{2}\right)\left(\sqrt{\frac{a+b}{2}}\right)
$$

and

$$
N_{3}(a, b)=\frac{a+\sqrt{a b}+b}{3} .
$$

The expression $N_{3}(a, b)$ is famous as Heron's mean. The inequalities (10) admit many non-negative differences. Bases on these difference the author [9; ?] proved 
the following result:

$$
D_{S A} \leq\left\{\begin{array}{l}
\frac{1}{3} D_{S H} \leq\left\{\begin{array}{l}
\frac{1}{2} D_{A H} \leq\left\{\begin{array}{l}
4 D_{N_{2} N_{1}} \leq \frac{4}{3} D_{N_{2} G} \leq D_{A G} \leq 4 D_{A N_{2}} \\
\frac{1}{2} D_{S G} \leq D_{A G}
\end{array}\right. \\
\frac{2}{3} D_{S N_{1}} \leq \frac{1}{2} D_{S G}
\end{array}\right. \\
\frac{4}{5} D_{S N_{2}} \leq 4 D_{A N_{2}} \\
\frac{3}{4} D_{S N_{3}} \leq \frac{2}{3} D_{S N_{1}}
\end{array}\right.
$$

where $D_{S A}=S-A, D_{S H}=S-H$, etc. Some applications of the inequalities (11) can be seen in [6], [7].

Combining (9) and (10), we have the following sequence of inequalities:

$$
P_{1} \leqslant P_{2} \leqslant P_{3} \leqslant H \leqslant P_{4} \leqslant G \leqslant N_{1} \leqslant N_{3} \leqslant N_{2} \leqslant A \leqslant\left(P_{5} \text { or } S\right) \leqslant P_{6} .
$$

The inequalities (12) admits many nonnegative differences such as

$$
D_{t p}(a, b)=b g_{t p}\left(\frac{a}{b}\right)=b\left[f_{t}\left(\frac{a}{b}\right)-f_{p}\left(\frac{a}{b}\right)\right],
$$

where

$$
g_{t p}(x)=f_{t}(x)-f_{p}(x), f_{t}(x) \geqslant f_{p}(x), \forall x>0 .
$$

More precisely, the expression (12) and the function $f:(0, \infty) \rightarrow \mathbb{R}$ given in (13) lead us to the following result:

$$
\begin{gathered}
f_{P_{1}}(x) \leqslant f_{P_{2}}(x) \leqslant f_{P_{3}}(x) \leqslant f_{H}(x) \leqslant f_{P_{4}}(x) \leqslant f_{G}(x) \leqslant f_{N_{1}}(x) \leqslant \\
\leqslant f_{N_{3}}(x) \leqslant f_{N_{2}}(x) \leqslant f_{A}(x) \leqslant\left(f_{P_{5}}(x) \text { or } f_{S}(x)\right) \leqslant f_{P_{6}}(x) .
\end{gathered}
$$

Equivalently,

$$
\begin{gathered}
\frac{x\left(x^{2}+1\right)}{x^{3}+1} \leqslant \frac{x(x+1)}{x^{2}+1} \leqslant \frac{x(\sqrt{x}+1)}{x^{3 / 2}+1} \leqslant \frac{2 x}{1+x} \leqslant \frac{4 x}{(\sqrt{x}+1)^{2}} \leqslant \\
\leqslant \sqrt{x} \leqslant\left(\frac{\sqrt{x}+1}{2}\right)^{2} \leqslant \frac{x+\sqrt{x}+1}{3} \leqslant\left(\frac{\sqrt{x}+1}{2}\right)\left(\sqrt{\frac{x+1}{2}}\right) \leqslant \\
\leqslant \frac{x+1}{2} \leqslant\left(\left(\frac{x+1}{\sqrt{x}+1}\right)^{2} \text { or } \sqrt{\frac{x^{2}+1}{2}}\right) \leqslant \frac{x^{2}+1}{x+1} .
\end{gathered}
$$

The inequalities appearing in (12) produces many nonnegative differences. In total, we have 77 differences. Some of them are not convex and some of them are convex. Some of them are equal to each other with some multiplicative constants such as

(i) $D_{P_{6} A}=D_{P_{6} H}=\frac{1}{2} D_{A H}$.

(ii) $D_{P_{5} P_{4}}=D_{A N_{3}}=\frac{2}{3} D_{A N_{1}}=\frac{1}{3} D_{A G}=2 D_{N_{3} N_{1}}=\frac{1}{2} D_{N_{3} G}=\frac{2}{3} D_{N_{1} G}$.

We leave to the readers to verify the convexity of the measures $D_{P_{6} N_{2}}, D_{P_{6} N_{3}}$, $D_{P_{6} N_{1}}, D_{P_{6} G}, D_{P_{6} P_{4}}, D_{P_{6} P_{2}}, D_{P_{6} P_{1}}, D_{P_{5} A}, D_{P_{5} N_{2}}, D_{P_{5} N_{3}}, D_{P_{5} N_{1}}, D_{P_{5} G}, D_{P_{5} H}$, $D_{A P_{4}}, D_{P_{6} S}, D_{S P_{4}}, D_{P_{6} S}$ and $D_{S P_{4}}$. While, the convexity of the difference of means 
INDER J. TANEJA

$D_{S A}, D_{S N_{2}}, D_{S N_{3}}, D_{S N_{1}}, D_{S G}, D_{S H}, D_{A N_{2}}, D_{A H}, D_{A G}, D_{N_{2} N_{1}}$ and $D_{N_{2} G}$ is already proved in Taneja [9; ?]. We observe that not all difference of means are convex [12].

In this paper our aim is to produce new inequalities for the difference of means arising due to inequalities given in (12). In another words we shall improve considerably the inequalities given in (11). For this we need first to know the convexity of the difference of means. In total, we have 77 differences. Some of them are equal to each other with some multiplicative constants. Some of them are not convex and some of them are convex.

\section{SEQUENCES OF INEQUALITIES}

In this section we shall bring sequence of inequalities based on the differences arising due to (12). The results given in this section are based on the applications of the following lemma [11]:

Lemma 2.1. Let $f_{1}, f_{2}: I \subset \mathbb{R}_{+} \rightarrow \mathbb{R}$ be two convex functions satisfying the assumptions:

(i) $f_{1}(1)=f_{1}^{\prime}(1)=0, f_{2}(1)=f_{2}^{\prime}(1)=0$;

(ii) $f_{1}$ and $f_{2}$ are twice differentiable in $\mathbb{R}_{+}$;

(iii) there exists the real constants $\alpha, \beta$ such that $0 \leqslant \alpha<\beta$ and

$$
\alpha \leqslant \frac{f_{1}^{\prime \prime}(x)}{f_{2}^{\prime \prime}(x)} \leqslant \beta, f_{2}^{\prime \prime}(x)>0,
$$

for all $x>0$ then we have the inequalities:

$$
\alpha \phi_{f_{2}}(a, b) \leqslant \phi_{f_{1}}(a, b) \leqslant \beta \phi_{f_{2}}(a, b),
$$

for all $a, b \in(0, \infty)$, where the function $\varphi_{(\cdot)}(a, b)$ is given by

$$
\phi_{f}(a, b)=a f\left(\frac{b}{a}\right), a, b>0 .
$$

Theorem 2.1. The following sequences of inequalities hold:

$$
\begin{aligned}
& \frac{1}{8} D_{P_{6} P_{1}} \leqslant \frac{1}{6} D_{P_{6} P_{2}} \leqslant D_{S A} \leqslant \frac{1}{3} D_{S H} \leqslant \frac{1}{2} D_{A H} \leqslant
\end{aligned}
$$

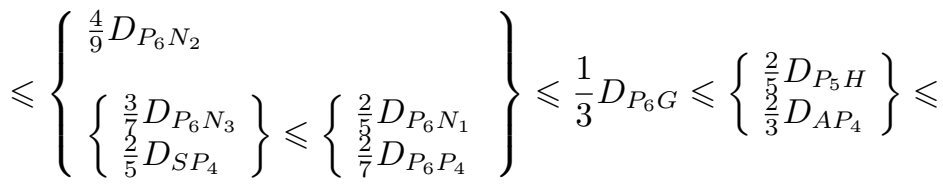

$$
\begin{aligned}
& \leqslant 4 D_{N_{2} N_{1}} \leqslant \frac{4}{3} D_{N_{2} G} \leqslant D_{A G} \leqslant 4 D_{A N_{2}} \leqslant \frac{2}{3} D_{P_{5} G} \leqslant \\
& \leqslant D_{P_{5} N_{1}} \leqslant \frac{6}{5} D_{P_{5} N_{3}} \leqslant \frac{4}{3} D_{P_{5} N_{2}} \leqslant 2 D_{P_{5} A} \text {, } \\
& D_{S A} \leqslant\left\{\begin{array}{c}
\frac{4}{5} D_{S N_{2}} \\
\frac{3}{4} D_{S N_{3}}
\end{array}\right\} \leqslant \frac{2}{3} D_{S N_{1}} \leqslant\left\{\begin{array}{c}
\frac{1}{3} D_{P_{6} G} \\
\frac{1}{2} D_{S G}
\end{array}\right\} \leqslant \frac{2}{5} D_{P_{5} H}
\end{aligned}
$$


and

$$
\left\{\begin{array}{l}
\frac{1}{8} D_{P_{6} P_{1}} \\
\frac{2}{13} D_{P_{5} P_{1}}
\end{array}\right\} \leqslant\left\{\begin{array}{l}
\frac{1}{6} D_{P_{6} P_{2}} \\
\frac{2}{9} D_{P_{5} P_{2}}
\end{array}\right\} \leqslant \frac{2}{7} D_{P_{5} P_{3}} \leqslant \frac{4}{9} D_{P_{6} N_{2}} \leqslant D_{P_{6} S} \leqslant D_{A G} .
$$

PROOF. We shall prove each part separately.

(1) For $\mathbf{D}_{\mathbf{P}_{6} \mathbf{P}_{1}} \leq \frac{4}{3} \mathbf{D}_{\mathbf{P}_{6} \mathbf{P}_{2}}$ : Let us consider a function $g_{P_{6} P_{1}-P_{6} P_{2}}(x)=f_{P_{6} P_{1}}^{\prime \prime}(x) / f_{P_{6} P_{2}}^{\prime \prime}(x)$, where $f_{P_{6} P_{1}}^{\prime \prime}(x)$ and $f_{P_{6} P_{2}}^{\prime \prime}(x)$ are as given by

$$
f_{P_{6} P_{1}}^{\prime \prime}(x)=\frac{2\left(x^{2}+1\right)\left(\begin{array}{c}
\left((x-1)^{2}-x\right)^{2}+x^{2}+ \\
+(x-1)^{4}+x(x-1)^{2}
\end{array}\right)+8 x^{3}}{\left(x^{3}+1\right)^{3}}
$$

and

$$
f_{P_{6} P_{2}}^{\prime \prime}(x)=\frac{2\left(x^{6}+15 x^{4}+16 x^{3}+15 x^{2}+1\right)}{(x+1)^{3}\left(x^{2}+1\right)^{3}},
$$

respectively. This gives $\beta=g_{P_{6} P_{1} P_{6} P_{2}}(1)=f_{P_{6} P_{1}}^{\prime \prime}(1) / f_{P_{6} P_{2}}^{\prime \prime}(1)=\frac{4}{3}$. Now we shall show that $\frac{4}{3} D_{P_{6} P_{2}}-D_{P_{6} P_{1}} \geq 0$. We can write $\frac{4}{3} D_{P_{6} P_{2}}-D_{P_{6} P_{1}}=$ $b f_{P_{6} P_{2}-P_{6} P_{1}}(a / b)$, where

$$
f_{P_{6} P_{2}-P_{6} P_{1}}(x)=\frac{4}{3} f_{P_{6} P_{2}}(x)-f_{P_{6} P_{1}}(x)=\frac{(x+1)(x-1)^{4}}{\left(x^{2}-x+1\right)\left(x^{2}+1\right)} .
$$

Since $f_{P_{6} P_{2} P_{6} P_{1}}(x)>0, \forall x>0, x \neq 1$, this proves the required result.

(2) For $\mathbf{D}_{\mathbf{P}_{6} \mathbf{P}_{2}} \leq 6 \mathbf{D}_{\mathbf{S A}}$ : Let us consider a function $g_{P_{6} P_{2}-S A}(x)=f_{P_{6} P_{2}}^{\prime \prime}(x) / f_{S A}^{\prime \prime}(x)$, where $f_{P_{6} P_{2}}^{\prime \prime}(x)$ and $f_{S A}^{\prime \prime}(x)$ are as given by

$$
f_{P_{6} P_{2}}^{\prime \prime}(x)=\frac{2\left(x^{6}+15 x^{4}+16 x^{3}+15 x^{2}+1\right)}{(x+1)^{3}\left(x^{2}+1\right)^{3}}
$$

and

$$
f_{S A}^{\prime \prime}(x)=\frac{1}{\left(x^{2}+1\right) \sqrt{2 x^{2}+2}},
$$

respectively. This gives $\beta=g_{P_{6} P_{1} S A}(1)=f_{P_{6} P_{2}}^{\prime \prime}(1) / f_{S A}^{\prime \prime}(1)=6$. Now we shall show that $6 D_{S A}-D_{P_{6} P_{2}} \geq 0$. We can write $6 D_{S A}-D_{P_{6} P_{2}}=$ $b f_{S A_{-} P_{6} P_{2}}(a / b)$, where

$$
f_{S A_{-} P_{6} P_{2}}(x)=6 f_{S A}(x)-f_{P_{6} P_{2}}(x)=\frac{k_{1}(x)}{\left(x^{2}+1\right)(x+1)},
$$

with

$$
k_{1}(x)=3 \sqrt{2 x+2}(x+1)\left(x^{2}+1\right)-\left(4 x^{4}+5 x^{3}+6 x^{2}+5 x+4\right) .
$$

Now we shall show that $k_{1}(x)>0, \forall x>0, x \neq 1$. Let us consider

$$
\begin{aligned}
h_{1}(x) & =\left[3 \sqrt{2 x+2}(x+1)\left(x^{2}+1\right)\right]^{2}-\left(4 x^{4}+5 x^{3}+6 x^{2}+5 x+4\right)^{2} . \\
& =(x-1)^{4}\left(2 x^{4}+4 x^{3}+3 x^{2}+4 x+2\right) .
\end{aligned}
$$




\section{INDER J. TANEJA}

Since $h_{2}(x)>0$ giving $k_{2}(x)>0, \forall x>0, x \neq 1$. This proves the required result.

Argument: Let $a$ and $b$ two positive numbers, i.e., $a>0$ and $b>0$. If $a^{2}-b^{2} \geq 0$, then we can conclude that $a \geq b$ because $a-b=\left(a^{2}-b^{2}\right) /(a+b)$. We have used this argument to prove $k_{1}(x) \geq 0, \forall x>0$. We shall use frequently this argument to prove the other parts.

Remark 2.1. According the parts 1 and 2, we observe that it is sufficient to write and show the nonnegativity of the expressions (19) and (20) for the other parts. The rest part of the proof follows on similar lines. Throughout, it is understood that $\forall x>$ $0, x \neq 1$.

(3) For $\mathbf{D}_{\mathrm{AH}} \leq \frac{8}{9} \mathbf{D}_{\mathbf{P}_{6} \mathrm{~N}_{2}}$ : In this case we have

$$
f_{P_{6} N_{2}-A H}(x)=\frac{8}{9} f_{P_{6} N_{2}}(x)-f_{A H}(x)=\frac{k_{2}(x)}{18(x+1)},
$$

with

$$
k_{2}(x)=7 x^{2}+18 x+7-4 \sqrt{2 x+2}(\sqrt{x}+1)(x+1) .
$$

Let us consider

$$
\begin{aligned}
h_{2}(x) & =\left(7 x^{2}+18 x+7\right)^{2}-[4 \sqrt{2 x+2}(\sqrt{x}+1)(x+1)]^{2} . \\
& =(\sqrt{x}-1)^{4}\left(17 x^{2}+4 x^{3 / 2}+38 x+4 \sqrt{x}+17\right) .
\end{aligned}
$$

(4) For $\mathbf{D}_{\mathrm{AH}} \leq \frac{6}{7} \mathbf{D}_{\mathrm{P}_{6} \mathrm{~N}_{3}}$ : In this case we have

$$
f_{P_{6} N_{3}-A H}(x)=\frac{6}{7} f_{P_{6} N_{3}}(x)-f_{A H}(x)=\frac{(\sqrt{x}-1)^{2}}{18(x+1)} .
$$

(5) For $\mathbf{D}_{\mathrm{AH}} \leq \frac{4}{5} \mathbf{D}_{\mathrm{SP}_{4}}$ : In this case we have

$$
f_{S P_{4}-A H}(x)=\frac{4}{5} f_{S P_{4}}(x)-f_{A H}(x)=\frac{k_{3}(x)}{10(x+1)(\sqrt{x}+1)^{2}},
$$

where

$$
\begin{aligned}
k_{3}(x)= & 4(x+1) \sqrt{2 x+2}(\sqrt{x}+1)^{2}- \\
& -\left(\begin{array}{c}
5 x^{3}+10 x^{5 / 2}+17 x^{2}+17 x+ \\
+10 x(\sqrt{x}-1)^{2}+10 \sqrt{x}+5
\end{array}\right) .
\end{aligned}
$$

Let us consider

$$
\begin{aligned}
h_{3}(x)= & {\left[4(x+1) \sqrt{2 x+2}(\sqrt{x}+1)^{2}\right]^{2}-} \\
& -\left(\begin{array}{l}
5 x^{3}+10 x^{5 / 2}+17 x^{2}+17 x+ \\
+10 x(\sqrt{x}-1)^{2}+10 \sqrt{x}+5
\end{array}\right)^{2} . \\
= & (\sqrt{x}-1)^{6}\left(\begin{array}{l}
7 x^{3}+70 x^{5 / 2}+201 x^{2}+ \\
+340 x^{3 / 2}+201 x+70 \sqrt{x}+7
\end{array}\right) .
\end{aligned}
$$


(6) For $\mathbf{D}_{\mathbf{P}_{6} \mathbf{N}_{3}} \leq \frac{14}{15} \mathbf{D}_{\mathbf{P}_{6} \mathbf{N}_{1}}$ : In this case we have

$$
f_{P_{6} N_{1} P_{6} N_{3}}(x)=\frac{14}{15} f_{P_{6} N_{1}}(x)-f_{P_{6} N_{3}}(x)=\frac{(\sqrt{x}-1)^{2}}{30(x+1)} .
$$

(7) For $\mathbf{D}_{\mathbf{P}_{6} \mathbf{N}_{3}} \leq \frac{2}{3} \mathbf{D}_{\mathbf{P}_{6} \mathbf{P}_{4}}$ : In this case we have

$$
f_{P_{6} P_{4}-P_{6} N_{3}}(x)=\frac{2}{3} f_{P_{6} P_{4}}(x)-f_{P_{6} N_{3}}(x)=\frac{\sqrt{x}(\sqrt{x}-1)^{4}}{3(\sqrt{x}+1)^{2}(x+1)} .
$$

(8) For $\mathbf{D}_{\mathbf{S P}_{4}} \leq \mathbf{D}_{\mathbf{P}_{6} \mathbf{N}_{1}}$ : In this case we have

$$
f_{P_{6} N_{1}-S P_{4}}(x)=f_{P_{6} N_{1}}(x)-f_{S P_{4}}(x)=\frac{k_{4}(x)}{4(x+1)(\sqrt{x}+1)^{2}},
$$

where

$$
\begin{gathered}
k_{4}(x)=3 x^{3}+4 x^{5 / 2}+9 x^{2}+9 x+4 x(\sqrt{x}-1)^{2}+4 \sqrt{x}+3 \\
-2 \sqrt{2 x^{2}+2}(x+1)(\sqrt{x}+1)^{2} .
\end{gathered}
$$

Let us consider

$$
\begin{aligned}
h_{4}(x)= & {\left[3 x^{3}+4 x^{5 / 2}+9 x^{2}+4 x(\sqrt{x}-1)^{2}+9 x+4 \sqrt{x}+3\right]^{2}-} \\
& -\left[2 \sqrt{2 x^{2}+2}(x+1)(\sqrt{x}+1)^{2}\right]^{2} . \\
= & (\sqrt{x}-1)^{4}\left(\begin{array}{l}
x^{3}(\sqrt{x}-2)^{2}+4 x^{3}+20 x^{5 / 2}+ \\
+78 x^{2}+20 x^{3 / 2}+4 x+(2 \sqrt{x}-1)^{2}
\end{array}\right) .
\end{aligned}
$$

(9) For $\mathbf{D}_{\mathbf{S P}_{4}} \leq \frac{5}{7} \mathbf{D}_{\mathbf{P}_{6} \mathbf{P}_{4}}$ : In this case we have

$$
f_{P_{6} P_{4}-S P_{4}}(x)=\frac{5}{7} f_{P_{6} P_{4}}(x)-f_{S P_{4}}(x)=\frac{k_{5}(x)}{14(x+1)(\sqrt{x}+1)^{2}},
$$

where

$$
\begin{aligned}
k_{5}(x)= & 10 x^{3}+20 x^{5 / 2}+26 x^{2}+26 x+20 \sqrt{x}+10 \\
& -7 \sqrt{2 x^{2}+2}(\sqrt{x}+1)^{2}(x+1) .
\end{aligned}
$$

Let us consider

$$
\begin{aligned}
h_{5}(x)= & \left(10 x^{3}+20 x^{5 / 2}+26 x^{2}+26 x+20 \sqrt{x}+10\right)^{2}- \\
& -\left[7 \sqrt{2 x^{2}+2}(\sqrt{x}+1)^{2}(x+1)\right]^{2} \\
= & 2(\sqrt{x}-1)^{4}\left(\begin{array}{l}
x^{4}+8 x^{7 / 2}+94 x^{3}+264 x^{5 / 2}+ \\
+386 x^{2}+264 x^{3 / 2}+94 x+8 \sqrt{x}+1
\end{array}\right) .
\end{aligned}
$$

(10) For $\mathbf{D}_{\mathbf{P}_{6} \mathbf{N}_{2}} \leq \frac{3}{4} \mathbf{D}_{\mathbf{P}_{6} \mathbf{G}}:$ In this case we have

$$
f_{P_{6} G_{-} P_{6} N_{2}}(x)=\frac{3}{4} f_{P_{6} G}(x)-f_{P_{6} N_{2}}(x)=\frac{k_{6}(x)}{4(x+1)},
$$


where

$$
k_{6}(x)=\sqrt{2 x+2}(\sqrt{x}+1)(x+1)-\left(x^{2}+1+3 x^{3 / 2}+3 \sqrt{x}\right) .
$$

Let us consider

$$
\begin{aligned}
h_{6}(x) & =[\sqrt{2 x+2}(\sqrt{x}+1)(x+1)]^{2}-\left(x^{2}+1+3 x^{3 / 2}+3 \sqrt{x}\right)^{2} \\
& =(\sqrt{x}-1)^{4}\left(x^{2}+2 x^{3 / 2}+x+2 \sqrt{x}+1\right) .
\end{aligned}
$$

(11) For $\mathbf{D}_{\mathbf{P}_{6} \mathrm{~N}_{1}} \leq \frac{5}{6} \mathbf{D}_{\mathbf{P}_{6} \mathrm{G}}$ : In this case we have

$$
f_{P_{6} G_{-} P_{6} N_{1}}(x)=\frac{5}{6} f_{P_{6} G}(x)-f_{P_{6} N_{1}}(x)=\frac{(\sqrt{x}-1)^{2}}{12(x+1)} .
$$

(12) For $\mathbf{D}_{\mathbf{P}_{6} \mathbf{P}_{4}} \leq \frac{7}{6} \mathbf{D}_{\mathbf{P}_{6} \mathrm{G}}$ : In this case we have

$$
f_{P_{6} G_{-} P_{6} P_{4}}(x)=\frac{7}{6} f_{P_{6} G}(x)-f_{P_{6} P_{4}}(x)=\frac{(\sqrt{x}-1)^{4}\left[(\sqrt{x}-1)^{2}+\sqrt{x}\right]}{12(x+1)} .
$$

(13) For $\mathbf{D}_{\mathbf{P}_{6} \mathrm{G}} \leq \frac{6}{5} \mathbf{D}_{\mathbf{P}_{5} \mathbf{H}}$ : In this case we have

$$
f_{P_{5} H_{-} P_{6} G}(x)=\frac{6}{5} f_{P_{5} H}(x)-f_{P_{6} G}(x)=\frac{(\sqrt{x}-1)^{4}\left[(\sqrt{x}-1)^{2}+\sqrt{x}\right]}{5(x+1)} .
$$

(14) For $\mathbf{D}_{\mathbf{P}_{6} \mathbf{G}} \leq \mathbf{2} \mathbf{D}_{\mathbf{A P}_{4}}$ : In this case we have

$$
f_{A P_{4}-P_{6} G}(x)=2 f_{A P_{4}}(x)-f_{P_{6} G}(x)=\frac{\sqrt{x}(\sqrt{x}-1)^{4}}{(\sqrt{x}+1)^{2}(x+1)} .
$$

(15) For $\mathbf{D}_{\mathbf{P}_{5} \mathrm{H}} \leq \mathbf{1 0 D}_{\mathbf{N}_{2} \mathrm{~N}_{1}}$ : In this case we have

$$
f_{N_{2} N_{1}-P_{5} H}(x)=10 f_{N_{2} N_{1}}(x)-f_{P_{5} H}(x)=\frac{k_{7}(x)}{2(\sqrt{x}+1)^{2}(x+1)},
$$

where

$$
\begin{aligned}
k_{7}(x)= & 5 \sqrt{2 x+2}(\sqrt{x}+1)^{3}(x+1)- \\
& -\left(7 x^{3}+20 x^{5 / 2}+37 x^{2}+32 x^{3 / 2}+37 x+20 \sqrt{x}+7\right) .
\end{aligned}
$$

Let us consider

$$
\begin{aligned}
h_{7}(x)= & {\left[5 \sqrt{2 x+2}(\sqrt{x}+1)^{3}(x+1)\right]^{2}-} \\
& -\left(7 x^{3}+20 x^{5 / 2}+37 x^{2}+32 x^{3 / 2}+37 x+20 \sqrt{x}+7\right)^{2} \\
= & (\sqrt{x}-1)^{4}\left(\begin{array}{l}
x^{4}+24 x^{7 / 2}+72 x^{3}+120 x^{5 / 2}+ \\
+126 x^{2}+120 x^{3 / 2}+72 x+24 \sqrt{x}+1
\end{array}\right) .
\end{aligned}
$$

(16) For $\mathrm{D}_{\mathrm{AP}_{4}} \leq 6 \mathrm{D}_{\mathrm{N}_{2} \mathrm{~N}_{1}}$ : In this case we have

$$
f_{N_{2} N_{1}-A P_{4}}(x)=6 f_{N_{2} N_{1}}(x)-f_{A P_{4}}(x)=\frac{k_{8}(x)}{2(\sqrt{x}+1)^{2}},
$$


SEQ. OF INEQ. AMONG DIFFERENCES OF GINI MEANS AND DIVERG. MEASURES

where

$$
k_{8}(x)=3 \sqrt{2 x+2}(\sqrt{x}+1)^{3}-2\left(2 x^{2}+7 x^{3 / 2}+6 x+7 \sqrt{x}+2\right) .
$$

Let us consider

$$
\begin{aligned}
h_{8}(x) & =\left[3 \sqrt{2 x+2}(\sqrt{x}+1)^{3}\right]^{2}-\left(4 x^{2}+14 x^{3 / 2}+12 x+14 \sqrt{x}+4\right)^{2} \\
& =2(\sqrt{x}-1)^{4}\left(x^{2}+2 x^{3 / 2}+2 \sqrt{x}+1\right) .
\end{aligned}
$$

(17) For $\mathbf{D}_{\mathrm{AN}_{2}} \leq \frac{1}{6} \mathbf{D}_{\mathrm{P}_{5} \mathrm{G}}:$ In this case we have

$$
f_{A N_{2}-P_{5} G}(x)=\frac{1}{6} f_{P_{5} G}(x)-f_{A N_{2}}(x)=\frac{k_{9}(x)}{12(\sqrt{x}+1)},
$$

where $k_{9}(x)=k_{8}(x)>0$.

(18) For $\mathbf{D}_{\mathbf{P}_{5} \mathrm{G}} \leq \frac{3}{2} \mathbf{D}_{\mathbf{P}_{5} \mathbf{N}_{1}}$ : In this case we have

$$
f_{P_{5} G_{-} P_{5} N_{1}}(x)=\frac{3}{2} f_{P_{5} G}(x)-f_{P_{5} N_{1}}(x)=\frac{(\sqrt{x}-1)^{2}}{8(x+1)} .
$$

(19) For $\mathbf{D}_{\mathbf{P}_{5} \mathbf{N}_{1}} \leq \frac{6}{5} \mathbf{D}_{\mathbf{P}_{5} \mathbf{N}_{3}}$ : In this case we have

$$
f_{P_{5} N_{3} P_{5} N_{1}}(x)=\frac{6}{5} f_{P_{5} N_{3}}(x)-f_{P_{5} N_{1}}(x)=\frac{(\sqrt{x}-1)^{2}}{20(x+1)} .
$$

(20) For $\mathbf{D}_{\mathbf{P}_{5} \mathrm{~N}_{3}} \leq \frac{10}{9} \mathbf{D}_{\mathbf{P}_{5} \mathbf{N}_{2}}$ : In this case we have

$$
f_{P_{5} N_{2}-P_{5} N_{3}}(x)=\frac{10}{9} f_{P_{5} N_{2}}(x)-f_{P_{5} N_{3}}(x)=\frac{k_{10}(x)}{18(\sqrt{x}+1)^{2}},
$$

where

$$
k_{10}(x)=2\left(4 x^{2}+9 x^{3 / 2}+14 x+9 \sqrt{x}+4\right)-5 \sqrt{2 x+2}(\sqrt{x}+1)^{3} .
$$

Let us consider

$$
\begin{aligned}
h_{10}(x) & =\left[2\left(4 x^{2}+9 x^{3 / 2}+14 x+9 \sqrt{x}+4\right)\right]^{2}-\left[5 \sqrt{2 x+2}(\sqrt{x}+1)^{3}\right]^{2} \\
& =2(\sqrt{x}-1)^{4}\left(7 x^{2}+22 x^{3 / 2}+32 x+22 \sqrt{x}+7\right) .
\end{aligned}
$$

(21) For $\mathbf{D}_{\mathbf{P}_{5} \mathbf{N}_{2}} \leq \frac{3}{2} \mathbf{D}_{\mathbf{P}_{5} \mathbf{A}}$ : In this case we have

$$
f_{P_{5} A_{-} P_{5} N_{2}}(x)=\frac{3}{2} f_{P_{5} A}(x)-f_{P_{5} N_{2}}(x)=\frac{k_{11}(x)}{4(\sqrt{x}+1)^{2}},
$$

where

$$
k_{11}(x)=\sqrt{2 x+2}(\sqrt{x}+1)^{3}-(x+1)(x+6 \sqrt{x}+1) .
$$

Let us consider

$$
\begin{aligned}
h_{11}(x) & =\left[\sqrt{2 x+2}(\sqrt{x}+1)^{3}\right]^{2}-[(x+1)(x+6 \sqrt{x}+1)]^{2} \\
& =(\sqrt{x}-1)^{4}(x+1)(x+4 \sqrt{x}+1) .
\end{aligned}
$$




\section{INDER J. TANEJA}

Parts 1-21 and the expression (11) together proves the sequences of inequalities appearing in (16).

(22) For $\mathbf{D}_{\mathrm{SN}_{2}} \leq \frac{5}{6} \mathbf{D}_{\mathbf{S N}_{1}}$ : In this case we have

$$
f_{S N_{1}-S N_{2}}(x)=\frac{5}{6} f_{S N_{1}}(x)-f_{S N_{2}}(x)=\frac{1}{24} \times k_{12}(x),
$$

where

$$
k_{12}(x)=6(\sqrt{x}+1) \sqrt{2 x+2}-\left[2 \sqrt{2 x^{2}+2}+5(\sqrt{x}+1)^{2}\right] .
$$

In order to prove $k_{12}(x)>0, \forall x>0, x \neq 1$, here we shall apply twice the argument given in part 2 . Let us consider

$$
\begin{aligned}
h_{12}(x)= & {[6(\sqrt{x}+1) \sqrt{2 x+2}]^{2}-2\left[\sqrt{2 x^{2}+2}+5(\sqrt{x}+1)^{2}\right]^{2} } \\
= & 39 x^{2}+41 x^{3 / 2}+3 \sqrt{x}(\sqrt{x}-1)^{2}+41 \sqrt{x}+39 \\
& -20(\sqrt{x}+1)^{2} \sqrt{2 x^{2}+2} .
\end{aligned}
$$

Applying again the same process over $h_{12}(x)$, we get

$$
\begin{aligned}
h_{12 a}(x)= & {\left[39 x^{2}+41 x^{3 / 2}+3 \sqrt{x}(\sqrt{x}-1)^{2}+41 \sqrt{x}+39\right]^{2} } \\
& -\left[20(\sqrt{x}+1)^{2} \sqrt{2 x^{2}+2}\right]^{2} \\
= & (\sqrt{x}-1)^{4}\left(721 x^{2}+3116 x^{3 / 2}+4806 x+3116 \sqrt{x}+721\right)^{2} .
\end{aligned}
$$

Since $h_{12 a}(x)>0$, giving $h_{12}(x)>0$. This proves $k_{12}(x)>0$.

(23) For $\mathbf{D}_{\mathbf{S N}_{1}} \leq \frac{1}{2} \mathbf{D}_{\mathbf{P}_{6} \mathbf{G}}:$ In this case we have

$$
f_{P_{6} G_{-} S N_{1}}(x)=\frac{1}{2} f_{P_{6} G}(x)-f_{S N_{1}}(x)=\frac{k_{13}(x)}{4(x+1)},
$$

where

$$
k_{13}(x)=3 x^{2}+2 x+3-2(x+1) \sqrt{2 x^{2}+2} .
$$

Let us consider

$$
\begin{aligned}
h_{13}(x) & =\left(3 x^{2}+2 x+3\right)^{2}-\left[2(x+1) \sqrt{2 x^{2}+2}\right]^{2} \\
& =(x-1)^{4} .
\end{aligned}
$$

(24) For $\mathbf{D}_{\mathrm{SG}} \leq \frac{4}{5} \mathbf{D}_{\mathbf{P}_{5} \mathbf{H}}:$ In this case, we have

$$
f_{P_{5} H_{-} S G}(x)=\frac{4}{5} f_{P_{5} H}(x)-f_{S G}(x)=\frac{k_{14}(x)}{4(x+1)},
$$

where

$$
\begin{aligned}
k_{14}(x)= & 2\left(4 x^{3}+5 x^{5 / 2}+11 x^{2}+3 x(\sqrt{x}-1)^{2}+11 x+5 \sqrt{x}+4\right)- \\
& -5(x+1)(\sqrt{x}+1)^{2} \sqrt{2 x^{2}+2}
\end{aligned}
$$


SEQ. OF INEQ. AMONG DIFFERENCES OF GINI MEANS AND DIVERG. MEASURES

Let us consider

$$
\begin{aligned}
h_{14}(x)= & {\left[2\left(4 x^{3}+5 x^{5 / 2}+11 x^{2}+3 x(\sqrt{x}-1)^{2}+11 x+5 \sqrt{x}+4\right)\right]^{2}-} \\
& -\left[5(x+1)(\sqrt{x}+1)^{2} \sqrt{2 x^{2}+2}\right]^{2} \\
= & 2(\sqrt{x}-1)^{4}\left(\begin{array}{l}
7 x^{4}+8 x^{7 / 2}+64 x^{3}+120 x^{5 / 2}+ \\
+242 x^{2}+120 x^{3 / 2}+64 x+8 \sqrt{x}+7
\end{array}\right) .
\end{aligned}
$$

(25) For $\mathbf{D}_{\mathbf{P}_{6} \mathrm{G}} \leq \frac{6}{5} \mathbf{D}_{\mathbf{P}_{5} \mathbf{H}}$ : In this case we have

$$
f_{P_{5} N_{3}-P_{5} N_{1}}(x)=\frac{6}{5} f_{P_{5} H}(x)-f_{P_{6} G}(x)=\frac{\left[(\sqrt{x}-1)^{2}+\sqrt{x}\right](\sqrt{x}-1)^{4}}{5(\sqrt{x}+1)^{2}(x+1)} .
$$

Parts 22-25 and the expression (11) together proves the sequences of inequalities appearing in (17).

(26) For $\mathbf{D}_{\mathbf{P}_{6} \mathbf{P}_{1}} \leq \frac{16}{9} \mathbf{D}_{\mathbf{P}_{5} \mathbf{P}_{2}}$ : In this case, we have

$$
\begin{aligned}
f_{P_{5} P_{2} P_{6} P_{1}}(x)= & \frac{16}{9} f_{P_{5} P_{2}}(x)-f_{P_{6} P_{1}}(x)= \\
= & \frac{(\sqrt{x}-1)^{4}\left(\begin{array}{l}
7 x^{5}+10 x^{9 / 2}+23 x^{4}+64 x^{7 / 2}+ \\
+142 x^{3}+180 x^{5 / 2}+142 x^{2}+ \\
+64 x^{3 / 2}+23 x+10 \sqrt{x}+7
\end{array}\right)}{9\left(x^{2}+1\right)\left(x^{3}+1\right)(\sqrt{x}+1)^{2}} .
\end{aligned}
$$

(27) For $\mathbf{D}_{\mathbf{P}_{5} \mathbf{P}_{1}} \leq \frac{13}{12} \mathbf{D}_{\mathbf{P}_{6} \mathbf{P}_{2}}$ : In this case, we have

$$
\begin{aligned}
f_{P_{6} P_{2} P_{5} P_{1}}(x)= & \frac{13}{12} f_{P_{6} P_{2}}(x)-f_{P_{5} P_{1}}(x)= \\
= & \frac{(\sqrt{x}-1)^{4}\left(\begin{array}{l}
x^{5}+30 x^{9 / 2}+89 x^{4}+152 x^{7 / 2}+ \\
+181 x^{3}+190 x^{5 / 2}+181 x^{2}+ \\
+152 x^{3 / 2}+89 x+30 \sqrt{x}+1
\end{array}\right)}{12\left(x^{2}+1\right)\left(x^{3}+1\right)(\sqrt{x}+1)^{2}} .
\end{aligned}
$$

(28) For $\mathbf{D}_{\mathbf{P}_{5} \mathbf{P}_{\mathbf{1}}} \leq \frac{13}{9} \mathbf{D}_{\mathbf{P}_{5} \mathbf{P}_{\mathbf{2}}}$ : In this case, we have

$$
\begin{aligned}
f_{P_{5} P_{2}-P_{5} P_{1}}(x)= & \frac{13}{9} f_{P_{5} P_{2}}(x)-f_{P_{5} P_{1}}(x)= \\
= & \frac{(\sqrt{x}-1)^{4}\left(\begin{array}{l}
4 x^{5}+16 x^{9 / 2}+44 x^{4}+88 x^{7 / 2}+ \\
+139 x^{3}+162 x^{5 / 2}+139 x^{2}+ \\
+88 x^{3 / 2}+16 \sqrt{x}+44 x+4
\end{array}\right)}{9\left(x^{2}+1\right)\left(x^{3}+1\right)(\sqrt{x}+1)^{2}} .
\end{aligned}
$$

(29) For $\mathbf{D}_{\mathbf{P}_{6} \mathbf{P}_{2}} \leq \frac{12}{7} \mathbf{D}_{\mathbf{P}_{5} \mathbf{P}_{3}}$ : In this case, we have

$$
\begin{aligned}
f_{P_{5} P_{3}-P_{6} P_{2}}(x) & =\frac{12}{7} f_{P_{5} P_{3}}(x)-f_{P_{6} P_{2}}(x)= \\
& =\frac{(\sqrt{x}-1)^{4}\left(\begin{array}{l}
5 x^{4}+x^{7 / 2}+17 x^{3}+22 x^{5 / 2}+ \\
+38 x^{2}+22 x^{3 / 2}+17 x+\sqrt{x}+5
\end{array}\right)}{7\left(x^{2}+1\right)\left(x^{3}+1\right)(\sqrt{x}+1)^{2}} .
\end{aligned}
$$


(30) For $\mathbf{D}_{\mathbf{P}_{5} \mathbf{P}_{2}} \leq \frac{9}{7} \mathbf{D}_{\mathbf{P}_{5} \mathbf{P}_{3}}$ : In this case, we have

$$
\begin{aligned}
f_{P_{5} P_{3} P_{5} P_{2}}(x) & =\frac{9}{7} f_{P_{5} P_{3}}(x)-f_{P_{5} P_{2}}(x)= \\
& =\frac{(\sqrt{x}-1)^{4}\left(\begin{array}{l}
2 x^{3}+6 x^{5 / 2}+16 x^{2}+ \\
+21 x^{3 / 2}+16 x+6 \sqrt{x}+2
\end{array}\right)}{7\left(x^{2}+1\right)(x-\sqrt{x}+1)(\sqrt{x}+1)^{2}} .
\end{aligned}
$$

(31) For $\mathbf{D}_{\mathbf{P}_{5} \mathbf{P}_{3}} \leq \frac{\mathbf{1 4}}{\mathbf{9}} \mathbf{D}_{\mathbf{P}_{\mathbf{6}} \mathbf{N}_{\mathbf{2}}}$ : In this case, we have

$$
\begin{aligned}
f_{P_{5} P_{3} P_{6} N_{2}}(x)= & \frac{14}{9} f_{P_{6} N_{2}}(x)-f_{P_{5} P_{3}}(x)= \\
= & \frac{(\sqrt{x}-1)^{4}\left(\begin{array}{l}
x^{6}+268 x^{11 / 2}+1143 x^{5}+782 x^{9 / 2}+ \\
+2597 x^{4}+2014 x^{7 / 2}+4478 x^{3}+ \\
+2014 x^{5 / 2}+2597 x^{2}+782 x^{3 / 2}+ \\
+1143 x+268 \sqrt{x}+1
\end{array}\right)}{18\left(x^{3}+1\right)(\sqrt{x}+1)^{2}} .
\end{aligned}
$$

(32) For $\mathbf{D}_{\mathbf{P}_{6} \mathbf{N}_{2}} \leq \frac{9}{4} \mathbf{D}_{\mathbf{P}_{6} \mathbf{S}}:$ In this case, we have

$$
f_{P_{6} S_{-} P_{6} N_{2}}(x)=\frac{9}{4} f_{P_{6} S}(x)-f_{P_{6} N_{2}}(x)=\frac{k_{15}(x)}{8(x+1)},
$$

where

$$
k_{15}(x)=2 \sqrt{2 x+2}(\sqrt{x}+1)(x+1)+10 x^{2}+10-9(x+1) \sqrt{2 x^{2}+2} .
$$

In order to prove $k_{15}(x)>0$, we shall apply twice the argument given in part 2. Let us consider

$$
\begin{aligned}
h_{15}(x)= & {\left[2 \sqrt{2 x+2}(\sqrt{x}+1)(x+1)+10 x^{2}+10\right]^{2}-} \\
& -\left[9(x+1) \sqrt{2 x^{2}+2}\right]^{2} \\
= & 40 \sqrt{2 x+2}(\sqrt{x}+1)\left(x^{2}+1\right)(x+1)- \\
& -\left(\begin{array}{l}
46 x^{4}+8(x+1)^{3}(\sqrt{x}-1)^{2}+ \\
+260 x^{3}+28 x^{2}+260 x+46
\end{array}\right) .
\end{aligned}
$$

Applying again over $h_{15}(x)$ the argument given in part 2, we get

$$
\begin{aligned}
h_{15 a}(x)= & {\left[40 \sqrt{2 x+2}(\sqrt{x}+1)\left(x^{2}+1\right)(x+1)\right]^{2}-} \\
& -\left(\begin{array}{l}
\left.46 x^{4}+8(x+1)^{3}(\sqrt{x}-1)^{2}+\right)^{2} \\
+260 x^{3}+28 x^{2}+260 x+46
\end{array}\right) \\
= & 4(\sqrt{x}-1)^{4}\left(\begin{array}{l}
71+2316 \sqrt{x}+11960 x^{5 / 2}+4090 x+ \\
+11960 x^{7 / 2}+11180 x^{3 / 2}+2316 x^{11 / 2}+ \\
+11180 x^{9 / 2}+12021 x^{4}+12021 x^{2}+ \\
+6004 x^{3}+4090 x^{5}+71 x^{6}
\end{array}\right) .
\end{aligned}
$$

Since $h_{15 a}(x)>0$ giving $h_{15}(x)>0$, and consequently, we have $k_{15}(x)>0$.

(33) For $\mathbf{D}_{\mathbf{P}_{6} \mathbf{S}} \leq \mathbf{D}_{\mathbf{A G}}$ : In this case, we have

$$
f_{A G_{-} P_{6} S}(x)=f_{A G}(x)-f_{P_{6} S}(x)=\frac{k_{16}(x)}{2(x+1)},
$$


with

$$
k_{16}(x)=\sqrt{2 x^{2}+2}(x+1)-\left[(x-1)^{2}+2(x+1) \sqrt{x}\right] .
$$

Now we shall show that $k_{16}(x)>0, \forall x>0, x \neq 1$. Let us consider

$$
\begin{aligned}
h_{16}(x) & =\left[\sqrt{2 x^{2}+2}(x+1)\right]^{2}-\left[(x-1)^{2}+2(x+1) \sqrt{x}\right]^{2} \\
& =(\sqrt{x}-1)^{6}(\sqrt{x}+1)^{2} .
\end{aligned}
$$

Parts 26-33 proves the sequences of inequalities appearing in (18).

Remark 2.2. In view of Theorem 2.1, we have the following improvement over the inequalities (11):

$$
D_{S A} \leqslant\left\{\begin{array}{l}
\frac{1}{3} D_{S H} \leqslant \frac{1}{2} D_{A H} \leqslant 4 D_{N_{2} N_{1}} \leqslant \frac{4}{3} D_{N_{2} G} \leqslant D_{A G} \leqslant 4 D_{A N_{2}} \\
\left\{\begin{array}{l}
\frac{4}{5} D_{S N_{2}} \\
\frac{3}{4} D_{S N_{3}}
\end{array}\right\} \leqslant \frac{2}{3} D_{S N_{1}} \leqslant \frac{1}{2} D_{S G}
\end{array} .\right.
$$

\subsection{Refinement Inequalities}

In this subsection, we shall present improvement over the inequalities given (12). Results obtained in Theorem 2.1, can be written in an individual form. Let us divide them in two two groups. The first one is with three measures and the second group is with four measures.

\section{Group 1}
(1) $P_{2} \leq \frac{P_{6}+3 P_{1}}{4}$;
(12) $N_{3} \leq \frac{P_{5}+5 N_{1}}{6}$;
(2) $\frac{2 S+H}{3} \leq A$;
(13) $N_{2} \leq \frac{P_{5}+9 N_{3}}{10}$;
(3) $\frac{P_{6}+14 N_{1}}{15} \leq N_{3}$;
(14) $A \leq \frac{P_{5}+2 N_{2}}{3}$;
(4) $\frac{P_{6}+2 P_{4}}{3} \leq N_{3}$;
(15) $\frac{S+4 N_{2}}{5} \leq A$;
(5) $S \leq \frac{5 P_{6}+2 P_{4}}{7}$;
(16) $\frac{S+3 N_{3}}{4} \leq A$;
(6) $\frac{P_{6}+3 G}{4} \leq N_{2}$;
(17) $\frac{S+5 N_{1}}{6} \leq N_{2}$;
(7) $\frac{P_{6}+5 G}{6} \leq N_{1}$;
(18) $\frac{S+8 N_{1}}{9} \leq N_{3}$;
(8) $G \leq \frac{P_{6}+6 P_{4}}{7}$;
(19) $\frac{S+3 G}{4} \leq N_{1}$;
(9) $\frac{2 N_{2}+G}{3} \leq N_{1}$;
(20) $P_{2} \leq \frac{9 P_{1}+4 P_{5}}{13}$;
(10) $N_{2} \leq \frac{3 A+G}{4}$;
(11) $N_{1} \leq \frac{P_{5}+2 G}{3}$;
(21) $P_{3} \leq \frac{2 P_{5}+7 P_{2}}{9}$;
(22) $S \leq \frac{5 P_{6}+4 N_{2}}{9}$.

\section{Group 2}
(1) $6 A+P_{6} \leq 6 S+P_{2}$;
(5) $S+N_{1} \leq P_{4}+P_{6}$;
(2) $9 A+8 N_{2} \leq 9 H+8 P_{6}$;
(6) $6 H+5 P_{6} \leq 6 P_{5}+5 G$;
(3) $9 A+6 N_{3} \leq 7 H+6 P_{6}$;
(7) $2 P_{4}+P_{6} \leq 2 A+G$;
(4) $5 \mathrm{~A}+4 \mathrm{P}_{4} \leq 5 \mathrm{H}+4 \mathrm{~S}$;
(8) $10 N_{1}+P_{5} \leq 10 N_{2}+H$; 
INDER J. TANEJA
(9) $A+6 N_{1} \leq P_{4}+6 N_{2}$;
(14) $13 P_{2}+12 P_{5} \leq 13 P_{6}+12 P_{1}$;
(10) $G+6 A \leq P_{5}+6 N_{2}$;
(15) $12 P_{3}+7 P_{6} \leq 12 P_{5}+7 P_{2}$
(11) $G+2 S \leq P_{6}+2 N_{1}$;
(16) $14 N_{2}+9 P_{5} \leq 14 P_{6}+9 P_{3}$;
(12) $4 H+5 S \leq 4 P_{5}+5 G$;
(17) $P_{6}+G \leq A+S$.
(13) $16 P_{2}+9 P_{6} \leq 16 P_{5}+9 P_{1}$;

Based on the inequalities appearing in Groups 1 and 2, we have the theorem giving the refinement over the inequalities appearing in (12).

Theorem 2.2. The following inequalities hold:

$$
\begin{aligned}
& G \leq \frac{P_{6}+6 P_{4}}{7} \leq\left\{\begin{array}{l}
\frac{P_{6}+5 G}{6} \\
\frac{S+3 G}{4}
\end{array}\right\} \leq \frac{2 N_{2}+G}{3} \leq N_{1} \leq\left\{\begin{array}{l}
\frac{10 N_{2}+H-P_{5}}{P_{4}+6 N_{2}-A} \\
6
\end{array}\right\} \leq \\
& \leq\left\{\begin{array}{l}
\left\{\begin{array}{l}
\frac{P_{5}+2 G}{3} \\
P_{4}+P_{6}-S
\end{array}\right\} \leq \frac{P_{6}+2 P_{4}}{3} \\
P_{4}+P_{6}-S\left\{\frac{P_{6}+14 N_{1}}{\frac{S+8 N_{1}}{9}}\right.
\end{array}\right\} \leq N_{3} \leq
\end{aligned}
$$

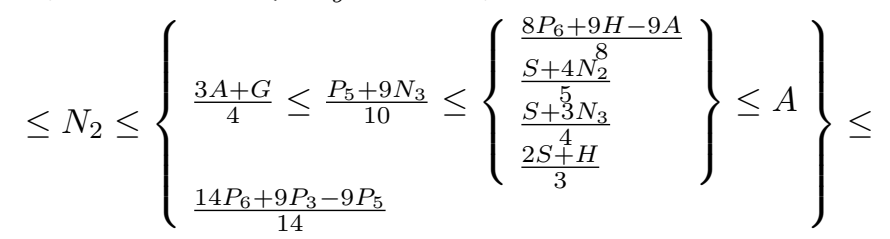

$$
\begin{aligned}
& \leq\left\{\frac{\frac{5 H+4 S-4 P_{4}}{P_{5}+6 N_{2}-G}}{6} \leq \frac{P_{5}+2 N_{2}}{3} \leq \frac{7 H+6 P_{6}-6 N_{3}}{7}\right\} \leq \\
& \leq\left\{\begin{array}{l}
\frac{4 H+5 S-5 G}{4} \leq P_{5} \\
S \leq \frac{P_{6}+2 N_{1}-G}{2} \leq \\
\frac{12 P_{5}+13 P_{2}-12 P_{1}}{13}
\end{array}\left\{\begin{array}{l}
\frac{2 P_{4}+5 P_{6}}{7} \\
\frac{4 N_{2}+5 P_{6}}{9}
\end{array}\right\} \leq P_{6} \leq\right. \\
& \leq\left\{\begin{array}{l}
A+S-G \\
\frac{6 P_{5}+5 G-6 H}{5} \\
2 A+G-2 P_{4}
\end{array}\right\} \leq\left\{\begin{array}{l}
\frac{12 P_{5}+7 P_{2}-12 P_{3}}{7} \\
6 S+P_{2}-6 A
\end{array}\right\} \leq \frac{16 P_{5}+9 P_{1}-16 P_{2}}{9}
\end{aligned}
$$

and

$$
P_{2} \leq\left\{\begin{array}{l}
\frac{P_{6}+3 P_{1}}{4} \leq N_{1} \leq \frac{P_{6}+3 G}{4} \leq \frac{S+5 N_{1}}{6} \leq N_{2} \\
\frac{4 P_{5}+9 P_{1}}{13} \leq N_{3} \leq \frac{P_{5}+5 N_{1}}{6} \leq A \leq \frac{3 I+2 P_{4}}{2} \leq\left\{\begin{array}{l}
P_{5} \leq \frac{T+2 A}{2} \leq \frac{3 J+16 G}{16} \\
S
\end{array}\right. \\
P_{3} \leq \frac{2 P_{5}+7 P_{2}}{9} \leq N_{1}
\end{array} .\right.
$$

The proof of the above theorem is based on the same techniques applied for the Theorem 2.1. This shall be dealt [13] elsewhere.

\section{CONNECTIONS WITH INFORMATION MEASURES}

Let

$$
\Gamma_{n}=\left\{P=\left(p_{1}, p_{2}, \ldots, p_{n}\right) \mid p_{i}>0, \sum_{i=1}^{n} p_{i}=1\right\}, n \geqslant 2
$$


be the set of all complete finite discrete probability distributions. For all $P, Q \in \Gamma_{n}$, the following inequalities are already proved by author [9]:

$$
\frac{1}{2} D_{A H} \leqslant I \leqslant 4 D_{N_{2} N_{1}} \leqslant \frac{4}{3} D_{N_{2} G} \leqslant D_{A G} \leqslant 4 D_{A N_{2}} \leqslant \frac{1}{8} J \leqslant T \leqslant \frac{1}{16} \Psi,
$$

where

$$
\begin{aligned}
& I(P \| Q)=\frac{1}{2}\left[\sum_{i=1}^{n} p_{i} \ln \left(\frac{2 p_{i}}{p_{i}+q_{i}}\right)+\sum_{i=1}^{n} q_{i} \ln \left(\frac{2 q_{i}}{p_{i}+q_{i}}\right)\right], \\
& J(P \| Q)=\sum_{i=1}^{n}\left(p_{i}-q_{i}\right) \ln \left(\frac{p_{i}}{q_{i}}\right), \\
& T(P \| Q)=\sum_{i=1}^{n}\left(\frac{p_{i}+q_{i}}{2}\right) \ln \left(\frac{p_{i}+q_{i}}{2 \sqrt{p_{i} q_{i}}}\right)
\end{aligned}
$$

and

$$
\Psi(P \| Q)=\sum_{i=1}^{n} \frac{\left(p_{i}-q_{i}\right)^{2}\left(p_{i}+q_{i}\right)}{p_{i} q_{i}} .
$$

The measures $I(P \| Q), J(P \| Q)$ and $T(P \| Q)$ are the respectively, the well-know Jensen-Shannon divergence, $J$-divergence and arithmetic and geometric mean divergence. The measure $\Psi(P \| Q)$ is symmetric chi-square divergence. For detailed study on these measures refer to Taneja [8; ?; ?]. Moreover, $D_{A H}(P \| Q)=\frac{1}{2} \Delta(P \| Q)$ and $D_{A G}(P \| Q)=h(P \| Q)$, where $\Delta(P \| Q)$ and $h(P \| Q)$ are the well-known triangular's and Hellinger's discriminations respectively.

In the theorem below we shall make connections of the classical divergence measures with the inequalities given in (16).

Theorem 3.1. The following inequalities hold:

$$
\begin{aligned}
& \left\{\begin{array}{l}
\frac{2}{5} D_{P_{5} H} \\
\frac{2}{3} D_{A P_{4}} \leqslant I
\end{array}\right\} \leqslant 4 D_{N_{2} N_{1}} \leqslant \frac{4}{3} D_{N_{2} G} \leqslant D_{A G} \leqslant 4 D_{A N_{2}} \leqslant \\
& \leqslant \frac{2}{3} D_{P_{5} G} \leqslant\left\{\begin{array}{l}
D_{P_{5} N_{1}} \leqslant \frac{6}{5} D_{P_{5} N_{3}} \leqslant \frac{4}{3} D_{P_{5} N_{2}} \leqslant 2 D_{P_{5} A} \\
\frac{1}{8}
\end{array}\right\} \leqslant T \leqslant \frac{1}{16} \Psi .
\end{aligned}
$$

PROOF. In view of the inequalities given in (16), it sufficient to show the inequalities $D_{A P_{4}} \leqslant \frac{3}{2} I, D_{P_{5} G} \leqslant \frac{3}{16} J$ and $D_{P_{5} A} \leqslant \frac{1}{2} T$. In each part of these three expressions we have logarithmic expressions. We shall apply a different approach to show them.

(i) For $\mathbf{D}_{\mathrm{AP}_{4}} \leqslant \frac{3}{2} \mathrm{I}$ : Let us consider

$$
g_{A P_{4-} I}(x)=\frac{f_{A P_{4}}^{\prime \prime}(x)}{f_{I}^{\prime \prime}(x)}=\frac{12 x(x+1)}{\sqrt{x}(\sqrt{x}+1)^{4}}, x \neq 1, x>0 .
$$

Calculating the first order derivative of the function $g_{A_{P_{4}} I}(x)$ with respect to $x$, 
one gets

$$
g_{A P_{4} I}^{\prime}(x)=-\frac{6\left(x^{3 / 2}-3 x+3 \sqrt{x}-1\right)}{\sqrt{x}(\sqrt{x}+1)^{5}}=-\frac{6(\sqrt{x}-1)^{3}}{\sqrt{x}(\sqrt{x}+1)^{5}} \begin{cases}>0, & x<1 \\ <0, & x>1\end{cases}
$$

In view of (25), we conclude that the function $g_{A P_{4} I}(x)$ is increasing in $x \in(0,1)$ and decreasing in $x \in(1, \infty)$, and hence

$$
\beta_{A P_{4_{-}} I}=\sup _{x \in(0, \infty)} g_{A P_{4_{-}} I}(x)=g_{A P_{4_{-} I}}(1)=\frac{3}{2} .
$$

By the application of Lemma 2.1 with (26) we get the required result.

(ii) For $\mathbf{D}_{\mathbf{P}_{\mathbf{5}} \mathrm{G}} \leqslant \frac{\mathbf{3}}{\mathbf{1 6}} \mathbf{J}$ : Let us consider

$$
g_{P_{5} G_{-} J}(x)=\frac{f_{P_{5} G}^{\prime \prime}(x)}{f_{J}^{\prime \prime}(x)}=\frac{3 \sqrt{x}\left(4 x^{3 / 2}+4 \sqrt{x}+x^{2}-2 x+1\right)}{4(x+1)(\sqrt{x}+1)^{4}} .
$$

Calculating the first order derivative of the function $g_{P_{5} G_{-} J}(x)$ with respect to $x$, one gets

$$
g_{P_{5} G_{-} J}^{\prime}(x)=-\frac{3(\sqrt{x}-1)^{3}\left(x^{2}+8 x^{3 / 2}+6 x+8 \sqrt{x}+1\right)}{8 \sqrt{x}(\sqrt{x}+1)^{5}(x+1)^{2}} \begin{cases}>0, & x<1 \\ <0, & x>1\end{cases}
$$

In view of (27), we conclude that the function $g_{P_{5} G_{-} J}(x)$ is increasing in $x \in(0,1)$ and decreasing in $x \in(1, \infty)$, and hence

$$
\beta_{P_{5} G_{-} J}=\sup _{x \in(0, \infty)} g_{P_{5} G_{-} J}(x)=g_{P_{5} G_{-} J}(1)=\frac{3}{2} .
$$

By the application of Lemma 2.1 with (28) we get the required result.

(iii) For $\mathbf{D}_{\mathbf{P}_{5} \mathbf{A}} \leqslant \frac{1}{2} \mathbf{T}$ : Let us consider

$$
g_{P_{5} A_{-} T}(x)=\frac{f_{P_{5} A}^{\prime \prime}(x)}{f_{T}^{\prime \prime}(x)}=\frac{2 \sqrt{x}\left(4 x^{3 / 2}+4 \sqrt{x}+x^{2}-6 x+1\right)(x+1)}{\left(x^{2}+1\right)(\sqrt{x}+1)^{4}} .
$$

Calculating the first order derivative of the function $g_{P_{5} A_{-} T}(x)$ with respect to $x$, one gets

$$
g_{P_{5} A_{-} T}^{\prime}(x)=-\frac{(\sqrt{x}-1)^{3}\left(\begin{array}{l}
8 \sqrt{x}\left(x^{2}+1\right)(\sqrt{x}-1)^{2}+ \\
+x^{4}+14 x^{3}+10 x^{2}+14 x+1
\end{array}\right)}{\sqrt{x}(\sqrt{x}+1)^{5}\left(x^{2}+1\right)^{2}} \begin{cases}>0, & x<1 \\
<0, & x>1\end{cases}
$$

In view of (29), we conclude that the function $g_{P_{5} A_{-} T}(x)$ is increasing in $x \in(0,1)$ and decreasing in $x \in(1, \infty)$, and hence

$$
\beta_{P_{5} A_{-} T}=\sup _{x \in(0, \infty)} g_{P_{5} A_{-} T}(x)=g_{P_{5} A_{-} T}(1)=\frac{1}{2} .
$$

By the application of Lemma 2.1 with (30) we get the required result.

Here we have referred Lemma 2.1, but its extension for the probability distributions is already proved in $[9 ;$ ?]. 
SEQ. OF INEQ. AMONG DIFFERENCES OF GINI MEANS AND DIVERG. MEASURES

\section{REFERENCES}

P. CZINDER and Z. PALES, Local monotonicity properties of two-variable Gini means and the comparison theorem revisited, J. Math. Anal. Appl., 301(2005), 427-438.

CHAO-PING CHEN, Asymptotic Representations for Stolarsky, Gini and the Generalized Muirhead Means, RGMIA COLLECTION, 11(4)(2008), 1-13.

C. GINI, Di una formula compressiva delle medie, Metron, 13(1938) 3-22.

D. H. LEHMER, On the compounding of certain means, J. Math. Anal. Appl., 36 (1971), 183-200.

J. SÁNDER, A Note on Gini Mean, General Mathematics, 12(4)(2004), 17-21.

H. N. SHI, J. ZHANG and DA-MAO LI, Schur-Geometric Convexity for Difference of Means, Applied Mathematics E-Notes, 10(2010), 275-284

S. SIMIĆ, A Simple Proof of Monotonicity for Stolarsky and Gini Means, Kragujevac J. Math, 32(2009), 75-79.

I.J. TANEJA, New Developments in Generalized Information Measures, Chapter in: Advances in Imaging and Electron Physics, Ed. P.W. Hawkes, 91(1995), 37-136.

I.J. TANEJA, On Symmetric and Nonsymmeric Divergence Measures and Their Generalizations, Chapter in: Advances in Imaging and Electron Physics, 138(2005), 177-250.

I.J. TANEJA, Refinement Inequalities Among Symmetric Divergence Measures, The Australian Journal of Mathematical Analysis and Applications, 2(1)(2005), Art. 8, pp. 1-23.

I.J. TANEJA, Refinement of Inequalities among Means, Journal of Combinatorics, Information and Systems Sciences, 31(2006), 357-378.

I.J. TANEJA, Inequalities among Differences of Gini Means and Divergence Measures, Available at: http://arxiv.org/abs/1105.5802.

I.J. TANEJA, Refinement of Gini-Means Inequalities and Connections with Divergence Measures, Available at: $h$ ttp://arxiv.org/abs/1111.5241.

Inder J. Taneja,

Departamento de Matemática

Universidade Federal de Santa Catarina

88.040-900 Florianópolis, SC, Brazil.

e-mail: ijtaneja@gmail.com

http://www.mtm.ufsc.br/ taneja

October May 2012 